УДК 517.532

\title{
Solution of Non-stationary Motion of Binary Mixture by Laplace Transformation
}

\author{
Nemat B. Darabi* \\ Institute of Mathematics and Computer Science \\ Siberian Federal University \\ Svobodny, 79, Krasnoyarsk, 660041 \\ Russia
}

Received 15.07.2018, received in revised form 02.01.2019, accepted 02.02.2019

In this paper is estimated a special solution for solving thermal diffusion equations, that describe motion of binary mixture in a flat layer. If Reynolds number is small, these equations are reduced to some easier inverse boundary problems. For solving these problems are used Laplace transformations. Temperatures are setted on the walls and velocity field is found. Analytical solution for stationary mode and numerical results for non-stationary regime are presented and is found, when boundary conditions stabilize with increasing time, then all velocity components and temperature go to stationary ones.

Keywords: Reynolds number, thermal diffusion equations, binary mixture and non-stationary flow. DOI: 10.17516/1997-1397-2019-12-2-240-248.

\section{Introduction}

Exact and approximate solutions of hydrodynamics equations are widely used for mathematical modeling of many processes in the chemical and petrochemical technology [1], including convection of mass processes and heat transfer, and various natural phenomena [2].

This paper deals with the unsteady motions of a binary mixture in a flat channel with solid fixed walls. Solution of the thermodiffusion convection equations is sought in a special form: one velocity component is a linear function along the length of channel, and the temperature and concentration are quadratic functions along this coordinate.

First time solutions for the stationary Navier-Stokes equations are considered by Hiemenz [3]. A review for similar type of exact solutions is available in [4]. The solution was used to describe the flow of a viscous fluid on the plane taking into account the adherence on it [5]. For moving plates nonstationary solutions Himenz was considered in [6]. In the works [7] and [8] given further development of the results [6], when distance between the plates varies according to a power function of time.

If in Himenz solution, pressure depends only on one spatial variable, then for the corresponding systems of equations it is necessary to solve direct problem [9]. In general, longitudinal pressure gradient further velocity, temperature and concentration fields are desired functions. Therefore, the initial-boundary value problem is reversible. In physical point of view, this means that for any mentioned pressure gradient, motion of mixture can not be realized. In this case, the problem is reduced to a series of one-dimensional inverse problems for parabolic equations

*nematdarabi@gmail.com

(C) Siberian Federal University. All rights reserved 
(thermal conductivity). For creeping motions (Re «1) are found exact solution of stationary problem and numerical results for non-stationary one and it is showed, that under certain conditions non-stationary results go to stationary with increasing time.

\section{Statement of problem}

Two-dimensional motion of a binary mixture in dimensionless variables, is described by the Oberbeck-Boussinesq's equations system [9]

$$
\begin{gathered}
u_{x}+v_{y}=0, \\
u_{t}+\operatorname{Re}\left(u u_{x}+v u_{y}\right)+p_{x}=u_{x x}+u_{y y}, \\
v_{t}+\operatorname{Re}\left(u v_{x}+v v_{y}\right)+p_{y}=v_{x x}+v_{y y}+\mathrm{G}(T+C), \\
T_{t}+\operatorname{Re}\left(u T_{x}+v T_{y}\right)=\frac{1}{\operatorname{Pr}}\left(T_{x x}+T_{y y}\right), \\
C_{t}+\operatorname{Re}\left(u C_{x}+v C_{y}\right)=\frac{1}{\mathrm{Sc}}\left[C_{x x}+C_{y y}-\varphi\left(T_{x x}+T_{y y}\right)\right],
\end{gathered}
$$

where $u(x, y, t), v(x, y, t)$ are the components of the velocity vector along the $x, y$ coordinate axes; $p(x, y, t)$ is the modified pressure (deviation from the hydrostatic pressure); $T(x, y, t)$, $C(x, y, t)$ are the deviations of temperature and concentration from their average constant values; $\operatorname{Re}=U_{0} h / \nu$ is Reynolds number; $U_{0}$ is the velocity characteristic of the flow, $\nu$ is kinematic viscosity, $h$ is the linear characteristic of the dimension; $\operatorname{Pr}=\nu / \chi$ is the Prandtl number, $\chi$ is the coefficient of thermal diffusivity; $\mathrm{Sc}=\chi / D$ is the Schmidt number, $D$ is the diffusion coefficient; $\psi=-\beta_{C} D^{T} /\left(\beta_{T} D\right)$ is the separation parameter, $\beta_{T}, \beta_{C}$ are the coefficients of thermal and concentration expansion of the mixture, $D^{T}$ is the thermal diffusion coefficient, in charge of the Soret effect; $\mathrm{G}=g \beta_{T} \Delta T h^{2} /\left(U_{0} \nu\right)$ is the analogue of Grashof number, $\Delta T$ is the characteristic of temperature difference in the mixture. All mentioned parameters are assumed to be constant.

The solution of (1) is sought in the next form

$$
\begin{gathered}
u(x, y, t)=U(y, t) x, \\
v(x, y, t)=V(y, t), \\
p(x, y, t)=W(y, t) \frac{x^{2}}{2}+D(y, t), \\
T(x, y, t)=A(y, t) x^{2}+B(y, t), \\
C(x, y, t)=M(y, t) x^{2}+N(y, t) .
\end{gathered}
$$

The velocity field (2) corresponds to the known solution [3] of the Navier-Stokes equations. As you can see from this equations, temperature includes expression $x^{2}$, that means it will be maximum (minimum) when function $A(y, t)$ takes negative (positive) amounts. Substitution expressions (2) in system of equations (1) leads to non-linear equations containing only functions, which depend on variables $y, t$. Suppose, that the motion is creeping $(\mathrm{R} \ll 1, \mathrm{G}=O(1))$, in addition, it happens in a flat layer with thickness (in dimensional variables $h$ )with solid fixed walls $y=0, y=1$. So, the above-mentioned equations become linear, and raises a number of 
initial-boundary value problems $0<y<1$,

$$
\begin{aligned}
& A_{t}=\frac{1}{\operatorname{Pr}} A_{y y} \\
& A(y, 0)=A_{0}(y), \quad A(0, t)=A_{1}(t), \quad A(1, t)=A_{2}(t) ; \\
& B_{t}=\frac{1}{\operatorname{Pr}}\left(B_{y y}+2 A\right) \text {, } \\
& B(y, 0)=B_{0}(y), \quad B(0, t)=B_{1}(t), \quad B(1, t)=B_{2}(t) ; \\
& M_{t}=\frac{1}{\mathrm{Sc}}\left(M_{y y}-\psi A_{y y}\right) \text {, } \\
& M(y, 0)=M_{0}(y),\left.\quad\left[M_{y}(y, t)-\psi A_{y}(y, t)\right]\right|_{y=0, y=1}=0 ; \\
& N_{t}=\frac{1}{\mathrm{Sc}}\left(N_{y y}+2 M-2 \psi A-\psi B_{y y}\right), \\
& N(y, 0)=N_{0}(y),\left.\quad\left[N_{y}(y, t)-\psi B_{y}(y, t)\right]\right|_{y=0, y=1}=0 \\
& U_{y y}-U_{t}=2 \mathrm{G} \int_{0}^{y}[A(z, t)+M(z, t)] d z+W_{0}(t), \\
& U(y, 0)=U_{0}(y), \quad U(0, t)=0, \quad U(1, t)=0 .
\end{aligned}
$$

Other unknown functions are defined as

$$
\begin{aligned}
& W(y, t)=2 \mathrm{G} \int_{0}^{y}[A(z, t)+M(z, t)] d z+W_{0}(t), \\
& D(y, t)=\mathrm{G} \int_{0}^{y}[B(z, t)+N(z, t)] d z+\int_{0}^{y}\left[V_{z z}(z, t)-V_{t}(z, t)\right] d z+D_{0}(t), \\
& V(y, t)=-\int_{0}^{y} U(z, t) d z .
\end{aligned}
$$

In (8) function $D_{0}(t)$ can be considered arbitrary, since in the initial problem (1), there are gradients of pressure. As regards to $W_{0}(t)$, which also appears in right side of equation (7), it is desired, thereby problems (3)-(7) will be reversible. An additional condition for $W_{0}(t)$ follows from the sticking conditions for vertical component of the velocity vector $V(y, t)$ at $y=1$

$$
\int_{0}^{1} U(z, t) d z=0
$$

There is similar condition on bottom wall $y=0$ by definition of $V(y, t)$ in the last equation (8).

Clearly, for smooth solutions of the problems (3)-(9), it's required to fulfill compatibility conditions

$$
\begin{gathered}
{\left.\left[M_{0 y}(y)-\psi A_{0 y}(y)\right]\right|_{y=0, y=1}=0,\left.\quad\left[N_{0 y}(y)-\psi B_{0 y}(y)\right]\right|_{y=0, y=1}=0} \\
\int_{0}^{1} U_{0}(z) d z=0, \quad U_{0}(0)=U_{0}(1)=0 .
\end{gathered}
$$

Boundary conditions for functions $A$ and $B$ correspond to specific temperatures on the walls $T(0, x, t)=A_{1}(t) x^{2}+B_{1}(t), T(1, x, t)=A_{2}(t) x^{2}+B_{2}(t)$ with known functions $A_{1}(t), A_{2}(t)$, $B_{1}(t), B_{2}(t)$. For functions $M$ and $N$ conditions on the walls mean lack of flow from them, 
namely $\left.\left[C_{y}-\psi T_{y}\right]\right|_{y=0, y=1}=0$. Also, it is possible to put heat isolation condition for one of the walls, for example $T_{y}=0$ at $y=0$. Therefore on this wall $C_{y}=0$.

Remark 1. Integrating from equation (5) taking into account the initial and boundary conditions, will be found

$$
\int_{0}^{1} M(z, t) d z=\int_{0}^{1} M_{0}(z) d z
$$

Remark 2. As it follows from the problem statement (3)-(9) functions $B(y, t), N(y, t)$ do not affect on velocity field, but they determine temperature field, concentration and pressure of binary mixture.

\section{Stationary flow}

Assume that $A_{j}(t)=A_{j}^{s}, B_{j}(t)=B_{j}^{s}$ with constants $A_{j}^{s}, j=1,2$. So, problems (3)-(9) have stationary solution

$$
\begin{gathered}
A^{s}(y)=A_{1}^{s}+\left(A_{2}^{s}-A_{1}^{s}\right) y, \\
B^{s}(y)=B_{1}^{s}+\left(B_{2}^{s}-B_{1}^{s}+\frac{A_{2}^{s}+2 A_{1}^{s}}{3}\right) y-A_{1}^{s} y^{2}-\left(A_{2}^{s}-A_{1}^{s}\right) \frac{y^{3}}{3}, \\
M^{s}(y)=\psi\left[A_{1}^{s}+\left(A_{2}^{s}-A_{1}^{s}\right) y\right]=\psi A^{s}(y), \\
N^{s}(y)=n^{s}+\psi\left[\left(B_{2}^{s}-B_{1}^{s}+\frac{A_{2}^{s}+2 A_{1}^{s}}{3}\right) y-A_{1}^{s} y^{2}-\left(A_{2}^{s}-A_{1}^{s}\right) \frac{y^{3}}{3}\right]=n^{s}+\psi\left(B^{s}(y)-B_{1}^{s}\right), \\
U^{s}(y)=\frac{1}{2} W_{0}^{s}\left(y^{2}-y\right)+\frac{\mathrm{G}(1+\psi)}{3}\left[A_{1}^{s}\left(y^{3}-y\right)+\frac{\left(A_{2}^{s}-A_{1}^{s}\right)}{4}\left(y^{4}-y\right)\right], \\
W_{0}^{s}=-\frac{\mathrm{G}(1+\psi)}{10}\left(7 A_{1}^{s}+3 A_{2}^{s}\right) .
\end{gathered}
$$

The vertical component of velocity $V^{s}(y)$ will be found by last equality (8) and is equal to

$$
V^{s}(y)=-\frac{1}{2} W_{0}^{s}\left(\frac{y^{3}}{3}-\frac{y^{2}}{2}\right)-\frac{\mathrm{G}(1+\psi)}{3}\left[A_{1}^{s}\left(\frac{y^{4}}{4}-\frac{y^{2}}{2}\right)+\frac{\left(A_{2}^{s}-A_{1}^{s}\right)}{4}\left(\frac{y^{5}}{5}-\frac{y^{2}}{2}\right)\right] .
$$

Another functions in (8) have the next representations

$$
\begin{gathered}
W^{s}(y)=W_{0}^{s}+\mathrm{G}(1+\psi)\left[2 A_{1}^{s} y+\left(A_{2}^{s}-A_{1}^{s}\right) y^{2}\right] \\
D^{s}(y)=\mathrm{G}\left\{\left(n^{s}+B_{1}^{s}\right) y+(1+\psi)\left[\left(B_{2}^{s}-B_{1}^{s}+\frac{A_{2}^{s}+2 A_{1}^{s}}{3}\right) \frac{y^{2}}{2}-\frac{A_{1}^{s} y^{3}}{3}-\left(A_{2}^{s}-A_{1}^{s}\right) \frac{y^{4}}{4}\right]\right\} .
\end{gathered}
$$

Constant $n^{s}$, which goes to the expression for $N^{s}(y)$ and $D^{s}(y)$, can be determined by setting the average concentration in dimensionless form $\left(C^{*}=\frac{\left(C-C_{\text {ave }}\right) \beta_{C}}{\Delta T \beta_{T}}\right)$ at $x=0$ :

$$
\left.\int_{0}^{1} C^{s}(x, y)\right|_{x=0} d z=0
$$

this gives

$$
n^{s}=-\frac{\psi}{2}\left(B_{2}^{s}-B_{1}^{s}+\frac{A_{1}^{s}+A_{2}^{s}}{6}\right)
$$




\section{Non-stationary motion}

Problems (3)-(9) can be reduced to problems for loaded equations (when $A(y, t), M(y, t)$ are known) to function $U(y, t)$, and then it will be reduced to an equation of the 2-nd relative to unknown $W_{0}(t)$, see., for example [10,11]. In these works, generally, unknown function of time includes a factor with known function of the variable $y$ and $t$. In this case, $W_{0}(t)$ goes to right side, additively, so is applied separation of variables method, in which basis functions along $y$ are solutions of problem as Sturm-Liouville, with integral conditions [12]. In this paragraph, using the specifics of the problems (3)-(9), will be applied the Laplace transformation method, which allows to receive quantitative characteristics of the motion for specific mixtures.

Suppose

$$
\hat{A}(y, p)=\int_{0}^{\infty} A(y, t) e^{-p t} d t
$$

is Laplace transformation of function $A(y, t)$. Definitions, properties and its domain of applicability see in [13]. In Laplace images problems (3)-(9) become boundary for the system of ordinary differential equation, the solution of which is in quadrature. Here are presented just formulas for basic unknowns $\hat{A}(y, p), \hat{M}(y, p), \hat{U}(y, p), \hat{W}_{0}(p)$ :

$$
\begin{aligned}
& \hat{A}(y, p)=\frac{1}{\operatorname{sh} \sqrt{p \operatorname{Pr}}}\left[\hat{A}_{2}(p)-\hat{A}_{1}(p) \operatorname{ch} \sqrt{p \operatorname{Pr}}+\sqrt{\frac{\operatorname{Pr}}{p}} \int_{0}^{1} A_{0}(z) \operatorname{sh} \sqrt{p \operatorname{Pr}}(y-z) d z\right] \times \\
& \times \operatorname{sh} \sqrt{p \operatorname{Pr}} y+\hat{A}_{1}(p) \operatorname{ch} \sqrt{p \operatorname{Pr}} y-\sqrt{\frac{\operatorname{Pr}}{p}} \int_{0}^{y} A_{0}(z) \operatorname{sh} \sqrt{p \operatorname{Pr}}(y-z) d z, \\
& \hat{M}(y, p)=\psi \hat{A}(y, p)+\sqrt{\frac{\mathrm{Sc}}{p}}\left[\int_{0}^{y} F(z, p) \operatorname{sh} \sqrt{p \mathrm{Sc}}(y-z) d z-\right. \\
& \left.-\frac{1}{\operatorname{sh} \sqrt{p \mathrm{Sc}}} \int_{0}^{1} F(z, p) \operatorname{ch} \sqrt{p \operatorname{Sc}}(1-z) d z \operatorname{ch} \sqrt{p \operatorname{Sc}} y\right] \\
& F(y, p)=\psi p \hat{A}(y, p)-M_{0}(y), \\
& \hat{U}(y, p)=\frac{\operatorname{sh} \sqrt{p} y}{\operatorname{sh} \sqrt{p}}\left[\frac{\hat{W}_{0}(p)}{p}(1-\operatorname{ch} \sqrt{p})-\frac{1}{\sqrt{p}} \int_{0}^{1} H(z, p) \operatorname{sh} \sqrt{p}(1-z) d z\right]+ \\
& +\frac{\hat{W}_{0}(p)}{p}(\operatorname{ch} \sqrt{p} y-1)+\frac{1}{\sqrt{p}} \int_{0}^{y} H(z, p) \operatorname{sh} \sqrt{p}(y-z) d z \\
& H(y, p)=2 \mathrm{G} \int_{0}^{y}[\hat{A}(z, p)+\hat{M}(z, p)] d z-U_{0}(y), \\
& \hat{W}_{0}(p)=\frac{1}{2 \operatorname{ch} \sqrt{p}-\sqrt{p} \operatorname{sh} \sqrt{p}-2}\left[\sqrt{p}(\operatorname{ch} \sqrt{p}-1) \int_{0}^{1} H(z, p) \operatorname{sh} \sqrt{p}(1-z) d z-\right. \\
& \left.-p \operatorname{sh} \sqrt{p} \int_{0}^{1} \int_{0}^{y} H(z, p) \operatorname{sh} \sqrt{p}(y-z) d z d y\right] .
\end{aligned}
$$

Suppose

$$
\lim _{t \rightarrow \infty} A_{j}(t)=A_{j}^{s},
$$

So using explicit expressions (11), are driven the next limit equalities

$$
\begin{aligned}
& \lim _{t \rightarrow \infty} A(y, t)=\lim _{p \rightarrow 0} p \hat{A}(y, p)=A^{s}(y), \\
& \lim _{t \rightarrow \infty} M(y, t)=\lim _{p \rightarrow 0} p \hat{M}(y, p)=M^{s}(y),
\end{aligned}
$$




$$
\begin{aligned}
& \lim _{t \rightarrow \infty} U(y, t)=\lim _{p \rightarrow 0} p \hat{U}(y, p)=U^{s}(y), \\
& \lim _{t \rightarrow \infty} W_{0}(t)=\lim _{p \rightarrow 0} p \hat{W}_{0}(p)=W_{0}^{s}
\end{aligned}
$$

where on the right sides, functions are stationary solutions in Section 2. Also, similar conclusions are valid for the functions $B(y, t), N(y, t)$. Finally, if equalities (12) are implemented, so with increasing time, solutions of the problems (3)-(9) go to stationary mode which are presented in Section 2.

\section{Numerical results}

Let us apply the numerical method of inversion of Laplace transformation to formulas in Section 3.

Required boundary conditions and initial conditions are supposed to

$$
\begin{gathered}
A_{1}(t)=1-4 e^{-\alpha t} \sin (\omega t), \\
M_{0}(y)=\psi(1-y), \quad A_{0}(y)=A_{2}(t)=0,
\end{gathered}
$$

and $\psi=0.1, \alpha=0.01, \omega=0.1, G=1$. It's obvious that, when this function takes amounts with minus sign so temperature has a maximum and vice versa. Equation (2) shows the relation between temperature and $A_{1}(t)$. For the better interpretation of results, desire functions will be shown at extremum points of function $A_{1}(t)$. So results for functions $A(y, t)$ and $M(y, t)$ are presented in Fig. 1.
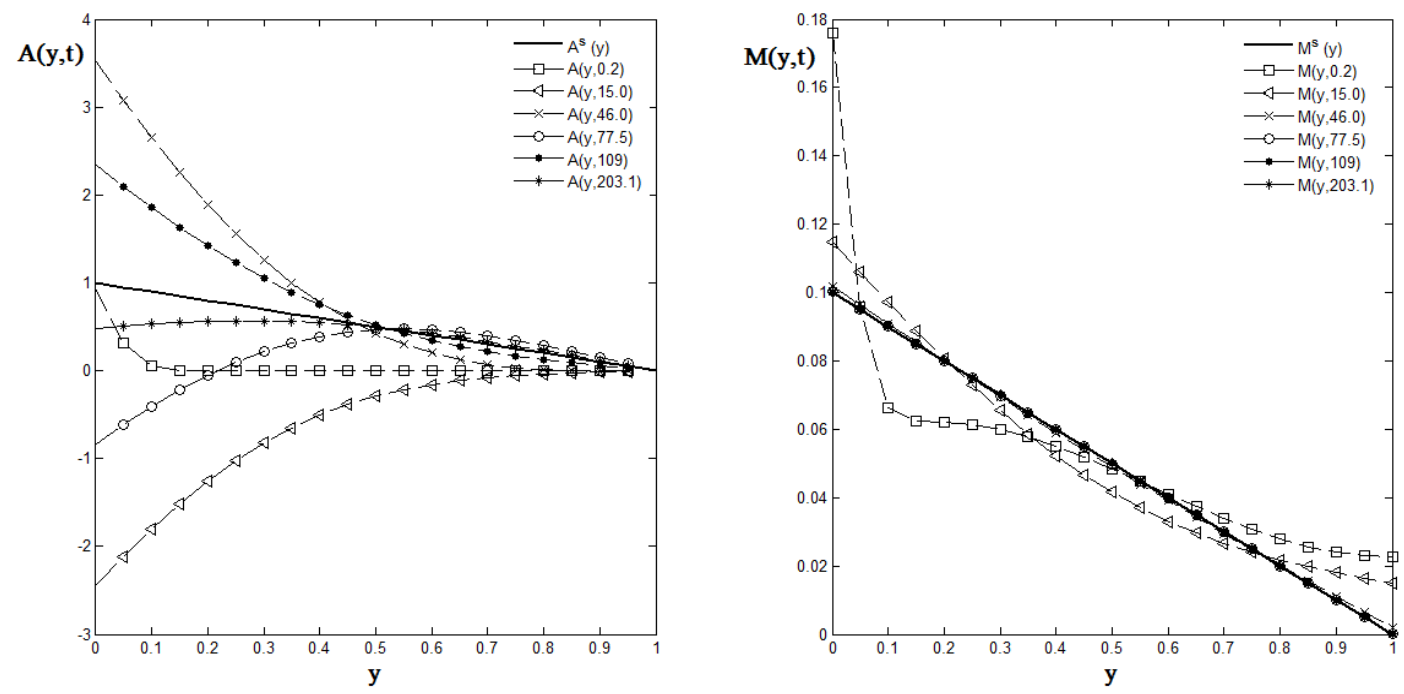

Fig. 1. Stationary and non-stationary results for $A(y, t)$ and $M(y, t)$

Here bold lines illustrate stationary solutions and non-bold lines are non-stationary ones. Each graph shows the results in extremum points for boundary condition $A_{1}(t)$. It is important about function $A(y, t)$, that all graphs, which indicate to minimum points in function $A_{1}(t)$, are placed above the stationary line whereas corresponding graphs for maximum points in $A_{1}(t)$, are down of it. About function $M(y, t)$, there isn't such relation between graphs and extremum points. 
Finally in Fig. 2 are presented the velocity components and it illustrates evolution of nonstationary regime to stationary one.
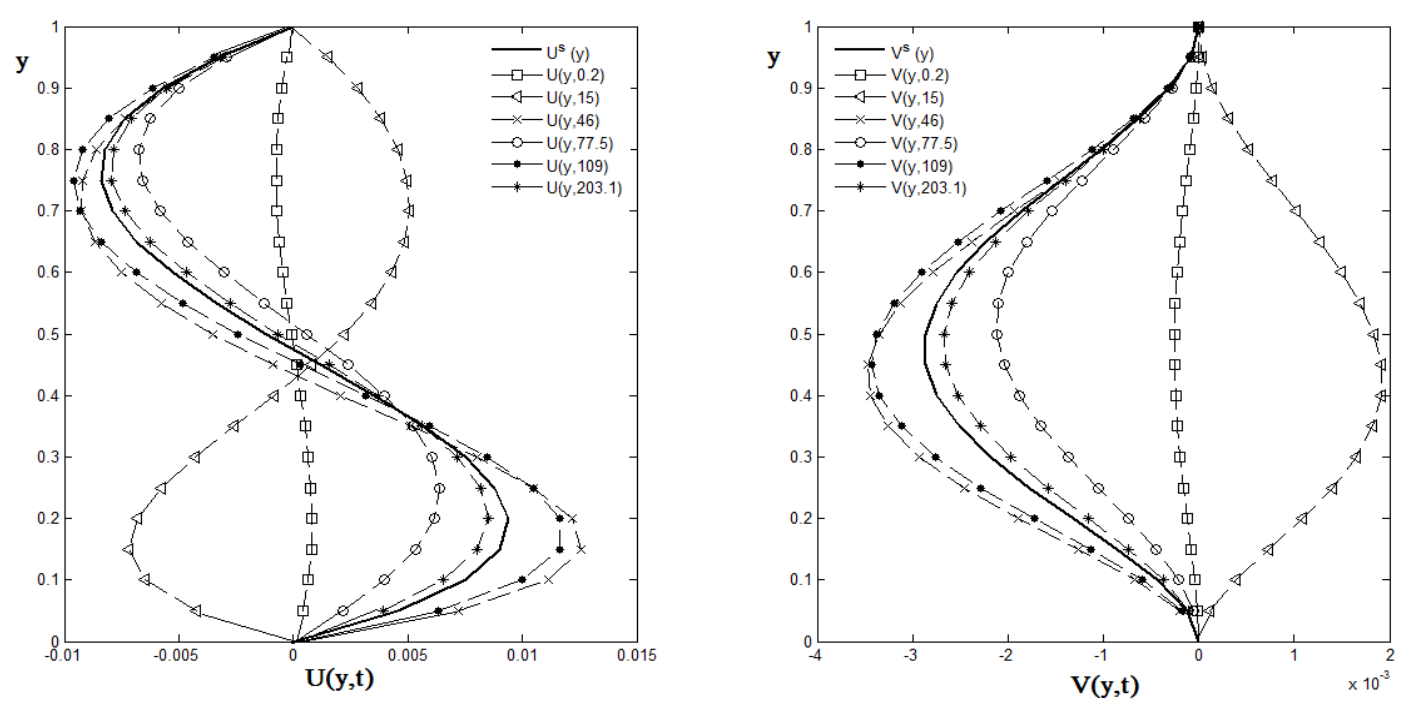

Fig. 2. Evolution of non-stationary motion of velocity components for $A_{1}(t)=1-4 e^{-\alpha t} \sin (\omega t)$

In according to numerical results (Fig. 2) non-stationary motion convergences to stationary. It was expected from theoretical concepts (12), so that when boundary conditions stabilize with increasing time, then all velocity components and temperature go to stationary ones.

Fig. 3 illustrates velocity components when $A_{1}(t)=2 \sin (0.01 t)$.
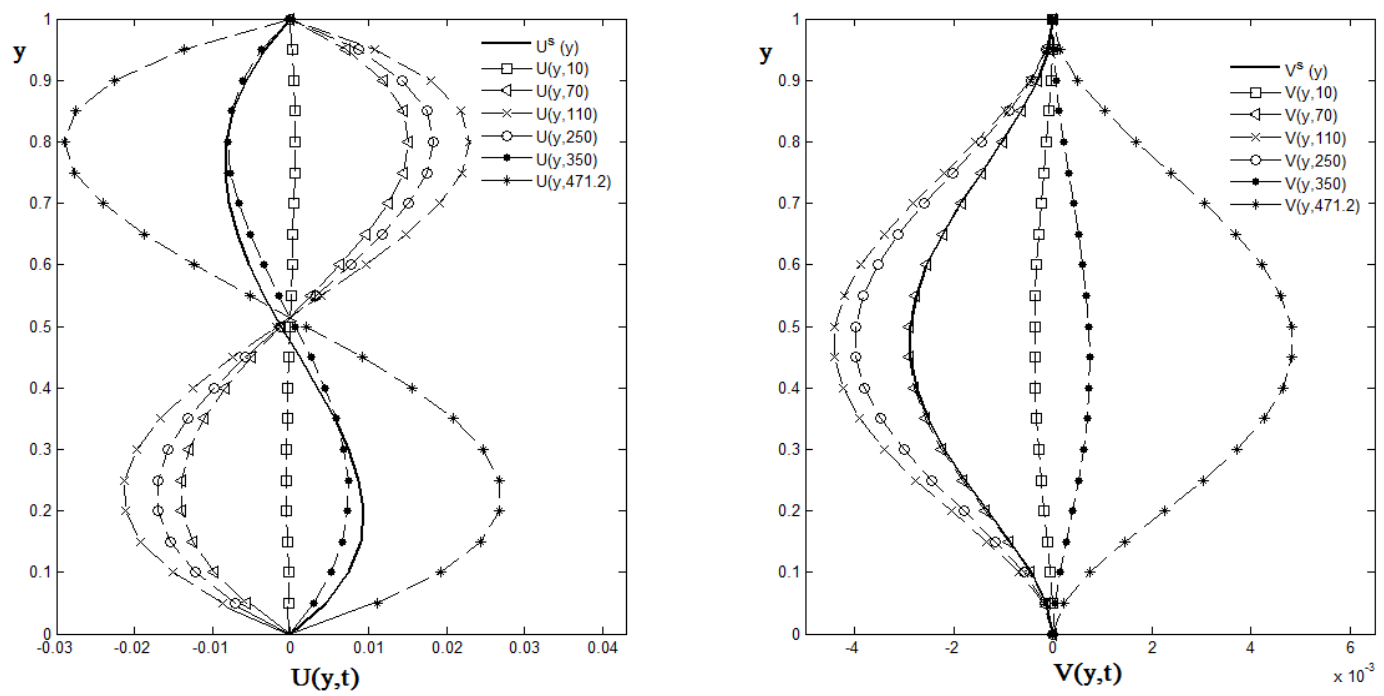

Fig. 3. Evolution of non-stationary motion of velocity components for $A_{1}(t)=2 \sin (0.01 t)$

Although non-stationary solution in some moments approximately coincides with stationary $(t \approx 350$ for $U(y, t)$ and $t \approx 70$ for $V(y, t))$, but for moments larger than these amounts the 
solutions diverge. Fig. 3 shows that time-dependent solution because of periodical boundary condition on bottom wall $\left(A_{1}(t)\right)$, fluctuates about stationary and there isn't any direct relation between them. This fact was extractable from theoretical points (12), because in this case $\lim _{t \rightarrow \infty} A_{1}(t)$ there isn't.

\section{References}

[1] A.D.Polyanin, A.M.Kutepov, A.V.Vyazmin, D.A.Kazenin, Hydrodynamics, Mass and Heat Transfer in Chemical Engineering, Taylor \& Francis, London, Vol. 14, 2001.

[2] V.K.Andreev, Y.A.Gaponenko, O.N.Goncharova, V.V.Pukhnachev, Mathematical models of convection, Walter de Gruyter, Berlin/Boston, 2012.

[3] K.Hiemenz, The boundary layer on a submerged straight circular cylinder in the uniform liquid flow, Dinglers Polytech Journal, 326(1911), 321-440.

[4] S.N.Aristov, D.V.Knyazev, A.D.Polyanin, Exact solutions of the Navier-Stokes equations with the linear dependence of velocity components on two space variables, Theoretical Foundations of Chemical Engineering, 5(2009), 547-566 (in Russian).

[5] J. F.Brady, A.Acrivos, Steady flow in a channel or tube with an accelerating surface velocity. An exact solution to the Navier-Stokes equations with reverse flow. journal of Fluid Mechanics, 112(1981), 127-150.

[6] R D.iabouchinsky, Quelques Considerations Sur les Mouvements Plans Rotationnels d 'un Liquide, C.R. Acad. Sci., 179(1924), 1133-1136.

[7] A.G.Petrov, Exact solution of the Navier-Stokes equations in a fluid layer between the moving parallel plates, Journal of Applied Mechanics and Technical Physics, 53(2012), 642-646.

[8] A.G.Petrov, Construction of solutions of the Navier-Stokes equations for the fluid layer between the moving parallel plates at low and moderate Reynolds numbers, Journal of Applied Mechanics and Technical Physics, 54(2013), 51-56.

[9] V.K.Andreev, N.L.Sobachkina, Movement of binary mixtures in flat and cylindrical domains, Siberian Federal University, Krasnoyarsk, 2012.

[10] A.I.Prilepko, D.G.Orlovsky, I. A.Vasin, Methods for Solving Inverse Problems in Mathematical Physics, Marsel Dekker, Inc., New-York, 1999.

[11] S.I.Kabanikhin, Inverse and Ill-Posed problems, Siberian Scientific Publishing House, Novosibirsk, 2009 (in Russian).

[12] V.K.Andreev, Unsteady 2D Motions a Viscous Fluid Described by Partially Invariant Solutions to the Navier-Stokes Equations, Journal of Siberian Federal University, 8(2015), no. 2, 140-147.

[13] M. A.Lavrent'ev, B.V.Shabat, Methods of theory of functions of complex variables, Nauka, Moscow, 1973 (in Russian). 


\section{Решение нестационарного движения бинарной смеси с помощью преобразования Лапласа}

Немат Б. Дараби Институт математики и фундаментальной информатики Сибирский федеральный университет Свободный, 79, Красноярск, 660041

Россия

В работе оценивается специальное решение для уравнений термодифбузии, описъвающих движение бинарной смеси в плоском слое. Если число Рейнолъдса мало, то эти уравнения сводятся $к$ некоторым простым обратным краевым задачам. Для решения таких задач используются преобразования Лапласа. На стенах устанавливаются температуры и определяется поле скоростей. Приведено аналитическое решение для стационарного режима и численные результатъ для нестационарного режима, когда граничные условия стабилизируются с увеличением времени, тогда все компоненты скорости и температура переходят к стационарным.

Ключевые слова: число Рейнольдса, уравнения термодиффузии, бинарная смесъ и нестационарное движение. 